\title{
Robotic Kinematic Simulation For Sewer Renovation
}

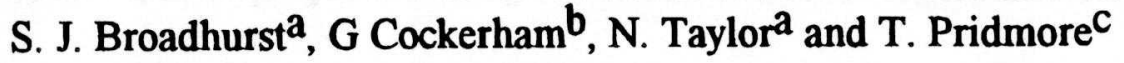 \\ aSchool of Construction, Sheffield Hallam University, City Campus, Sheffield, S1 \\ 1WB, United Kingdom \\ bSchool of Engineering, Sheffield Hallam University, City Campus, Sheffield, S1 \\ 1WB, United Kingdom \\ 'School of Engineering Information Technology, Sheffield Hallam University, City \\ Campus, Sheffield, S1 1WB, United Kingdom
}

\begin{abstract}
Research into the development of robotic modules is discussed in the context of costeffective, small bore, non-man entry (NME) sewer renovation. With an associated sensing system previously reported [1], interest herein is centred upon suitably robust task kinematics and control, where predictive modelling is implemented employing the GRASP robotic simulation software package.
\end{abstract}

\section{Sewer Renovation Problem}

Currently, approximately $95 \%$ of the UK population are connected to the main drainage system, which is the highest proportion by world standards. Some $96 \%$ of the approx. $250,000 \mathrm{~km}$ of existing sewers are of NME classification $(<1 \mathrm{~m}$ diameter) and limited access to these must be obtained directly from manhole locations, or from within man-entry systems with which they intersect. Sewers inherently transport media which is hazardous to the health of any operative associated with, or working within, this environment.

In the early 1980's an estimated $2500-5000$ sewer collapses were occurring in Britain every year [2,3]; the problems of sewer dereliction are in general attributable to their Victorian origins and ensuing, infrequent maintenance resulting in an unknown degree of general disrepair $[4,5]$. Collapses are largely dealt with on the basis of reactive maintenance. Major repairs require trenching procedures or the use of trenchless 'hard' liners. However, for NME sewers, 'soft' Insituform relining technology is widely employed. CCTV surveys assess the problem; this is followed by relining whereupon sled/tractor mounted cutters are then used for purposes of reconnection of the NME sewer to the subsidiary (and even smaller) 'lateral pipes' through which waste enters the NME sewer. This is an awkward task and final CCTV surveys are employed to assess the overall renovation. Lateral reconnections by remote techniques are currently prone to human error, reducing the effectiveness of the process. Notwithstanding the 
current availability of expensive, semi-robotic systems employing umbilicals, this research programme is centred upon the study and implementation of cost-effective, task-specific, fire-and-forget robotic modules designed about proprietary tractor units.

These modules comprise a CPU, work-head, kinematic structure and environmental sensing system, the configuration of which is constrained by the task environment. It is believed that the hazardous and confined nature of this environment will force new developments in each of the four key components. Whilst this confined nature constrains permissible robotic activities, advantages include known 'world' geometry [6].

\section{Sensor Study}

Initial efforts have been centred upon the development of a novel computer vision sensing facility [1, 7]; this is surely logical, with sensing preceding controlled action. Through the use of direct and reflected illumination, coupled with a single fixed camera, a method for lateral detection based on specified visual decision criteria has been developed. It should be noted that re-location of the later lining-obscured lateral intersection orifice, a prerequisite for the kinematic and control factors discussed below, is of necessity to be a non-visual procedure.

\section{Task Kinematic Model}

NME lateral connections to the main sewer are most commonly aligned at either $45^{\circ}$ or $90^{\circ}$, with connections generally occurring centreline-to-centreline; laterals may also possess inclination at the intersection. A $90^{\circ}$ intersection model pre-supposing a horizontal lateral lie at the joint itself, as shown in Figure 1, will be considered for present purposes; note that $R_{m}$ and $R_{1}$ denote $N M E$ sewer and lateral pipe radii respectively.

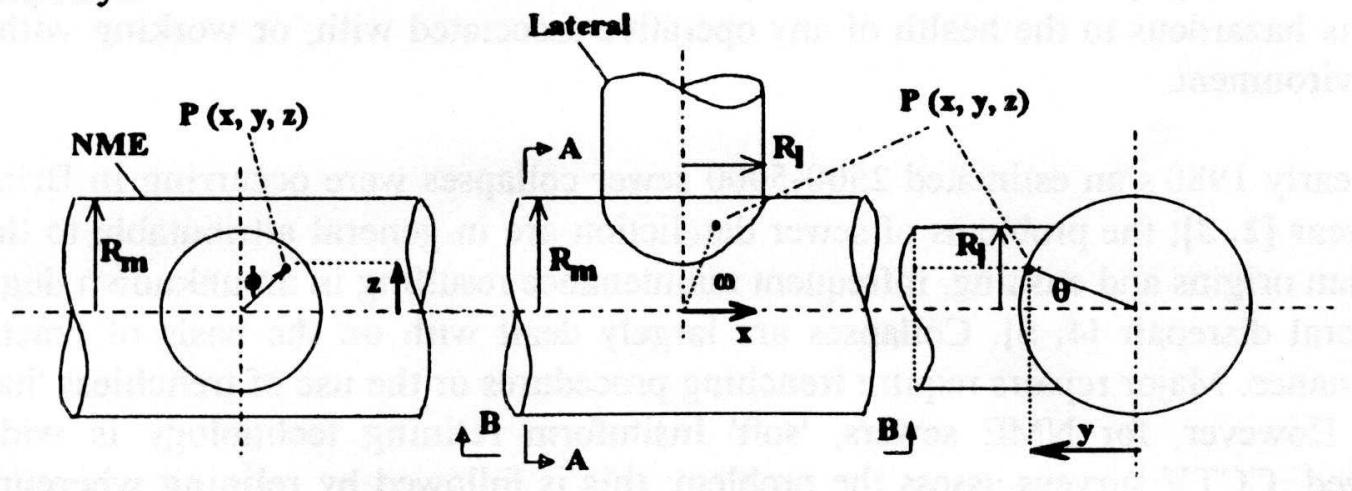

Elevation B-B

Plan

Crose-Section A-A

(nb: spatial coordinates $x, y, z$ relative to origin at centrelines' intersection)

Figure 1: Sewer/Lateral Intersection Model 
Predictive modelling is implemented employing GRASP, a 3D (off-line) robotic simulation software package [8]. GRASP permits the user to interactively produce robot designs for operation within task specific environments such as the sewer environment illustrated in isometric form in Figure 2. Robots with up to 6 Degrees Of Freedom (DOF's) task/arm movements can be specified, employing translational and rotational joints for which performance envelopes must be declared. The animation of robot models provides analysis/assessment of their suitability to the task requirements including, for example, the compatibility of performance envelopes with environment constraints.

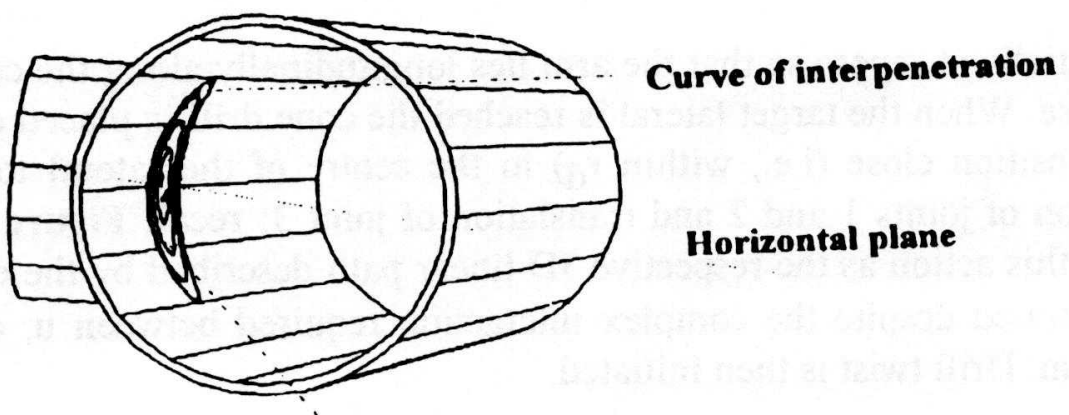

These loci are circles deformed onto a cylindrically curved surface termed 'curves of intersection'

Fig 2: GRASP Generated Sewer Model, Showing Curves Of Intersection

As shown in Figure 3, the task of lateral reconnection is considered in terms of two orthogonal arm rotations ( $\omega$ and $\theta$ ) and one arm extension movement ( $u$ ) in addition to the end-effector taking the form of a cone twist drill of radius $\mathbf{r}_{\mathbf{d}}$; a proprietary tractor unit has also been acquired and modelled in GRASP for prototype development purposes.

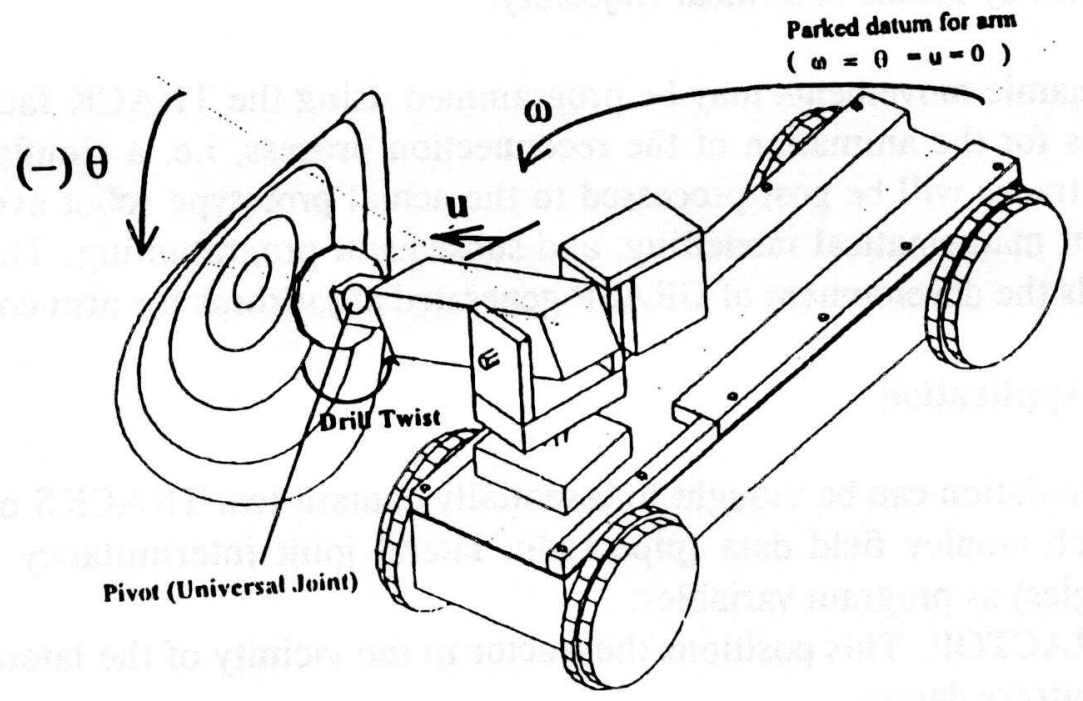

Figure 3: GRASP Generated Tractor/Arm Model 
The kinematic procedures required to reconnect the lateral, post-lining, can now be implemented.

Rotational movements are provided for in both the horizontal plane, $\omega$ (joint 1 ), and in the vertical plane, $\theta$ (joint 2). Together with arm extension u (joint 3 ), these kinematic parameters must provide for reaching the furthest part of the lateral to be cut. A spring tensioned universal joint mechanism is provided at the end-effector to allow the cone drill to pivot about its attachment point so as to align the cutting surface of the drill with the lateral wall to produce least abrasion of the surface.

The arm is initially at repose so that the arm lies longitudinally along the centre of the tractor unit base. When the target lateral is reached the cone drill is placed on the liner surface at a position close (i.e., within $r_{d}$ ) to the centre of the lateral to be drilled through rotation of joints 1 and 2 and translation of joint 3; recall Figure 3. GRASP shows well in this action as the respective 3D linear path described by the end effector is simply generated despite the complex interaction required between $u, \omega$ and $\theta$ to achieve this aim. Drill twist is then initiated.

It is assumed that the required action of the conical drill will be to grind the liner away rather than cut out an actual solid section, which could otherwise cause an obstruction elsewhere in the sewer. Following emplacement therefore, thrust is applied (u) and the drill grinds away a curve of intersection path. Upon completion of the path the cone drill continues grinding whilst stepping out radially a distance not greater than $r_{d}$. Thereupon, this curve of intersection/step grinding cycle is continued until the last step reaches $\leq R_{1}-r_{d}$ whereupon a final circular grinding loop is made. Typical values of $\mathrm{R}_{1}$ are 150,225 and $300 \mathrm{~mm}$ and are to be acquired from council records or previous survey data. At this juncture, drill twist is deactivated and the arm withdrawn and returned to repose by means of a linear trajectory.

In GRASP, dynamic movements may be programmed using the TRACK facility. This facility provides for the animation of the reconnection process, i.e. a simulation. It is envisaged that tracks will be post-processed to the actual prototype robot avoiding the need for explicit mathematical modelling, and subsequent programming. The analysis therefore affords the development of GRASP generated algorithms for arm control.

\section{Task Control Application}

The GRASP simulation can be thought to essentially contain two TRACKS or grouped operations which employ field data (pipe radii, lateral joint intermittancy distances, intersection angles) as program variables;

- MOVE_TRACTOR; This positions the tractor in the vicinity of the lateral relative to some arbitrary datum 


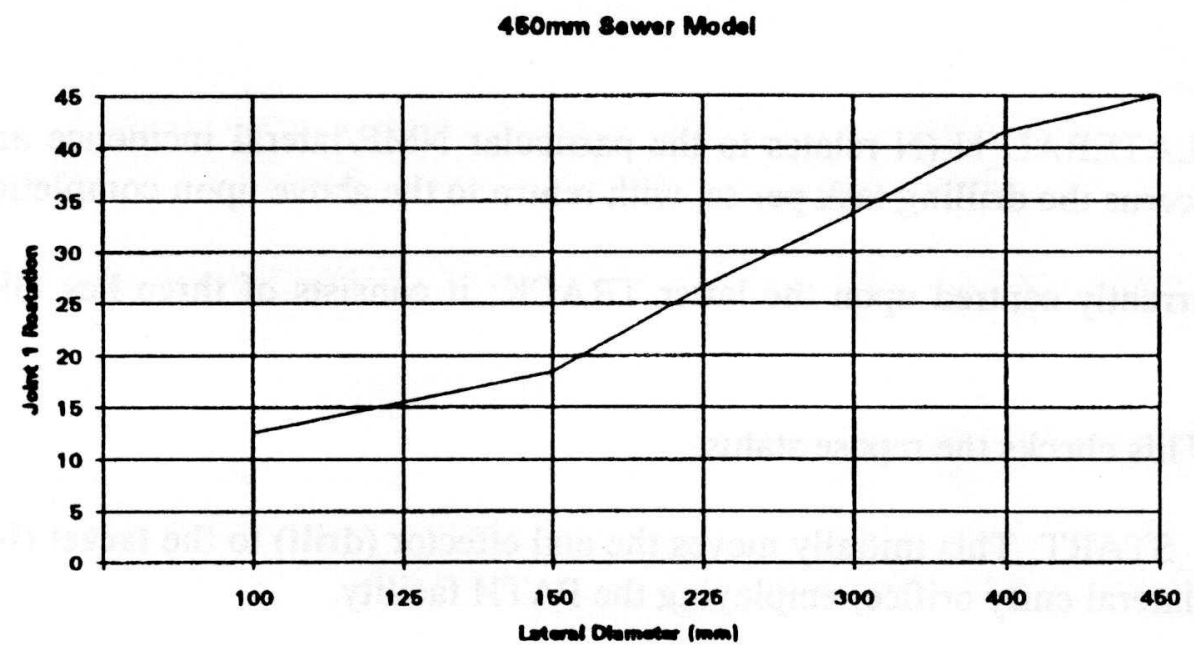

Figure 4: Joint 1 Rotation ( $\omega)$

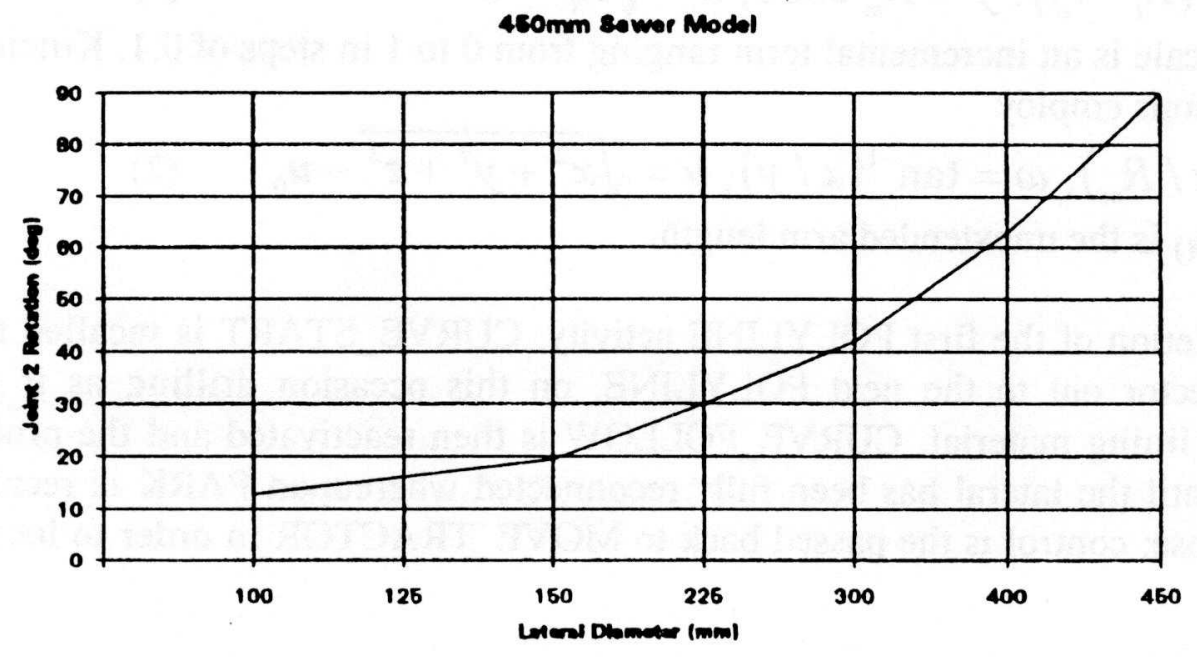

Figure 5: Joint 2 Rotation $(\theta)$ 
and

- DRILL_LATERAL_N (N relates to the particular NME/lateral incidence angle); This concerns the drilling task per se, with return to the above upon completion.

Interest is currently centred upon the latter TRACK; it consists of three key GRASP procedures;

- PARK; This checks the repose status.

- CURVE_START; This initially moves the end effector (drill) to the target (liningcovered lateral entry orifice) employing the PATH facility.

- CURVE_FOLLOW; A POLYLINE facility is used to grind out a curve of intersection path. This requires input from a $C$ programme based upon the requisite geometry as indicated in Figure 1. Typical location $\mathrm{x}, \mathrm{y}, \mathrm{z}$ is given by

$z=$ scale $^{*}\left(R_{l}-r_{d}\right), y=R_{m} \cos \theta, x=\sqrt{R_{l}^{2}-z^{2}}$

where scale is an incremental term ranging from 0 to 1 in steps of 0.1 . Kinematic expressions employ

$\theta=\sin ^{-1}\left(z / R_{m}\right), \omega=\tan ^{-1}(x / y), u=\sqrt{x^{2}+y^{2}+z^{2}}-u_{0}$ where $u_{0}$ is the unextended arm length.

Upon completion of the first POLYLINE activity, CURVE_START is recalled to step the end effector out to the next POLYLINE, on this occasion drilling as it moves through the lining material. CURVE_FOLLOW is then reactivated and the procedure continues until the lateral has been fully reconnected whereupon PARK is recalled to achieve repose; control is the passed back to MOVE_TRACTOR in order to locate the next lateral.

Figures 4, 5 and 6 show appropriate robotic design characteristics for representative field data (previously noted) 


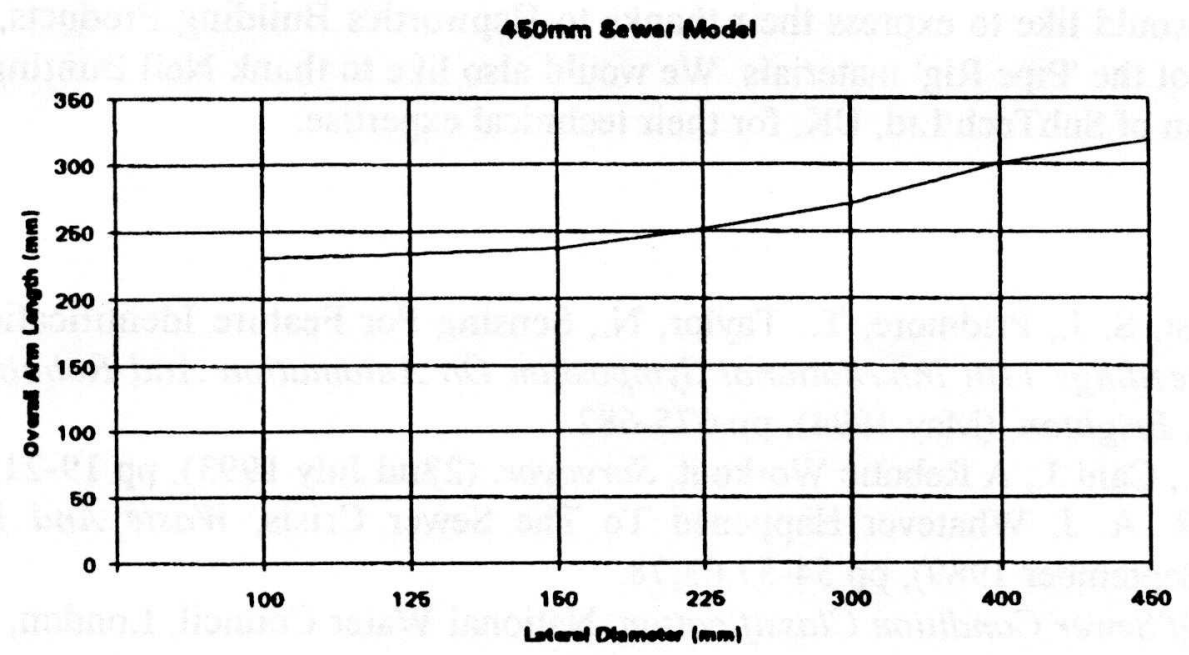

Figure 6: Overall Arm Length $\left(u+u_{o}\right)$.

\section{Comments}

The robot detailed above is currently being constructed; the drives for the robot arm and their power requirements are currently under investigation. The tractor unit weighs in the region of $40 \mathrm{kgf}$, robot stability during liner cutting is not expected to be a problem, and is powered by a $60 \mathrm{~V} \mathrm{DC}$ motor. Work is underway to adapt the existing power train so that when the tractor unit is stationary, having achieved its target location, drive will be deactivated from the tractor wheels through a simple clutch mechanism and directed towards powering joint $1(\omega)$. Joint $2(\theta)$, will be powered by a stepper motor, which will also provide the power for the drill.

The GRASP simulation presently models joint 3 (u) as a powered translational movement, allowing the robot arm to extend so as to be able to reach the furthest point on the lateral profile; note Figure 6. However, it may prove feasible to adopt a sprung slide way which will allow this joint movement to be attained directly, without the need for any form of further powered drive (and weight).

A $10 \mathrm{~m}$ long, $450 \mathrm{~mm}$ internal diameter, concrete sewer pipe testing rig has been constructed. Both $90^{\circ}$ and $45^{\circ}$ lateral intersections are included within the rig which will be used to test the accuracy of the robotic drilling arm later in 1995. It is envisaged that the experimentation will involve the integration of the computer vision and robotic drilling system with a view to the on-line monitoring of the lateral reconnections for 'quality assurance'. Incorporation of a system for the digital determination of the distance travelled by the robot along the sewer bottom is also required noting that actual tractor motion involves excursions from this path. 


\section{Acknowledgements}

The authors would like to express their thanks to Hepworth's Building Products, UK, for provision of the 'Pipe Rig' materials. We would also like to thank Neil Bunting and Russ Chapman of SubTech Ltd, UK, for their technical expertise.

\section{References}

[1] Broadhurst, S. J., Pridmore, T., Taylor, N., Sensing For Feature Identification In Sewers, Proceedings 11th International Symposium On Automation And Robotics In Construction, Brighton, (May 1994), pp 675-682.

[2] Russell A., Cant J., A Robotic Workout, Surveyor, (22nd July 1993), pp 19-21.

[3] Arthur, R. A. J, Whatever Happened To The Sewer Crisis, Waste And Water Treatment, (September 1989), pp 54-57,63,78.

[4] Manual Of Sewer Condition Classification, National Water Council, London, May 1980).

[5] Sewers And Water Mains - A National Assessment, National Water Council, London, (June 1977).

[6] Taylor, N., Draper, R., Broadhurst, S. J., Mallinder, P. A., Static And Kinematic Limit States Of Masonry Vaults, The Centenary Year Bridge Conference Proceedings, University Of Wales College Of Cardiff, Elesvier, 1994, pp 181-186.

[7] Broadhurst, S. J., Pridmore, T. P., Taylor, N., Three Dimensional Description Of Sewer Laterals Via Reflective Photometric Stereo, 12th International Symposium On. Automation And Robotics In Construction, Warsaw, Submitted For Publication.

[8] Naghdy F., Baldwin S. D., Simulation Of Locomotion On GRASP, Automation In Construction, 1, (1), (May 1992), pp 83-96. 